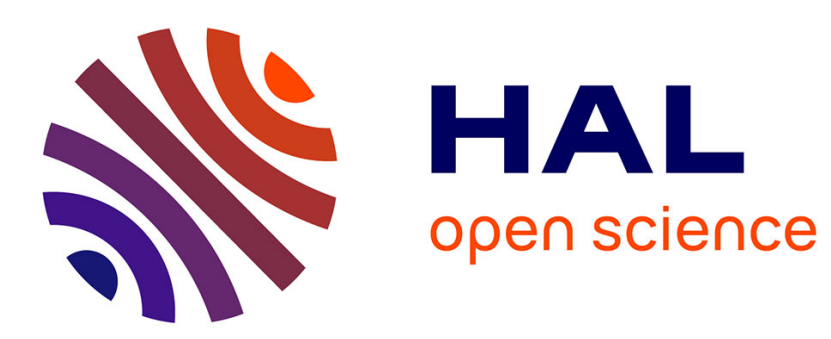

\title{
Dynamique des systèmes mécaniques discrets avec liaisons unilatérales parfaites
}

Patrick Ballard

\section{To cite this version:}

Patrick Ballard. Dynamique des systèmes mécaniques discrets avec liaisons unilatérales parfaites. Comptes rendus de l'Académie des sciences. Série IIb, Mécanique, physique, astronomie, 1999, 327 (10), pp.953-958. 10.1016/S1287-4620(00)87003-9 . hal-00111629

\section{HAL Id: hal-00111629 \\ https://hal.science/hal-00111629}

Submitted on 2 Apr 2018

HAL is a multi-disciplinary open access archive for the deposit and dissemination of scientific research documents, whether they are published or not. The documents may come from teaching and research institutions in France or abroad, or from public or private research centers.
L'archive ouverte pluridisciplinaire HAL, est destinée au dépôt et à la diffusion de documents scientifiques de niveau recherche, publiés ou non, émanant des établissements d'enseignement et de recherche français ou étrangers, des laboratoires publics ou privés. 


\title{
Dynamique des systèmes mécaniques discrets avec liaisons unilatérales parfaites
}

\author{
Patrick BALLARD
}

Laboratoire de mécanique des solides, École polytechnique, 91128 Palaiseau cedex, France

\begin{abstract}
La dynamique des systèmes mécaniques discrets avec liaisons unilatérales parfaites est formulée dans un cadre très général. Le problème d'évolution correspondant est étudié. On montre l'existence et l'unicité d'une solution maximale sous l'hypothèse que toutes les données sont analytiques. Une condition suffisante pour assurer que la solution maximale est définie pour tout temps est également donnée.
\end{abstract}

mécanique analytique / dynamique / liaisons unilatérales / impact / inclusion différentielle

\section{The dynamics of discrete mechanical systems with perfect unilateral constraints}

\begin{abstract}
The dynamics of discrete mechanical systems with perfect unilateral constraints is formulated in a very general setting. We study the resulting evolution problem. The existence and uniqueness of a maximal solution is proved under the hypothesis that the data are analytic. We also provide a sufficient condition to ensure that the maximal solution is defined for all time.

analytical mechanics / dynamics / unilateral constraints / impact / differential inclusion
\end{abstract}

\section{Abridged English Version}

DEFINITION 1. - A discrete mechanical system is:

- A Hausdorff smooth connected manifold $Q$ which is called the configuration space of the discrete mechanical system. Its dimension $d$ is the number of degrees of freedom. The tangent bundle $T Q$ of $Q$ is called the phase space. A point $q$ of $Q$ is a configuration of the system and a point of $T Q$ a state of the system. $T^{*} Q$ denotes the cotangent bundle, $I_{Q}: T Q \rightarrow Q$ and $\Pi_{Q}^{*}: T^{*} Q \rightarrow Q$ the natural projections. $T_{q} Q$ is, as usual, the tangent space at $q$ and, to designate an element $v$ of $T Q$, we use the redundant notation $(q, v)$ where $q=I_{Q}(q, v)$.

- A riemannian metric on $Q$ denoted by $(.,)_{q}$. The mapping $K$ defined by formula (1) is the kinetic energy of the system. 
- A real interval $I$ and a smooth mapping $f: T Q \times I \rightarrow T^{*} Q$ such that : $\forall(q, v) \in T Q, \forall t \in I$, $\Pi_{Q}^{*}(f(q, v ; t))=\Pi_{Q}(q, v)=q$. The mapping $f$ is called virtual power of internal, external and inertial efforts acting on the system or, in short, the efforts mapping. We denote by $\langle.,\rangle_{q}$ the local duality product on $T_{q}^{*} Q \times T_{q} Q$ and by $\mathrm{b}$ (and \# $=\mathrm{b}^{-1}$ its inverse) the isomorphism of vector bundles from $T Q$ onto $T^{*} Q$ canonically associated with the riemannian metric on $Q$.

In the case where no supplementary constraints are added to the system, the Fundamental Principle of Dynamics asserts that any motion of the system is of class $C^{2}$ and has to satisfy the equation of motion (2), where $\frac{\mathrm{D}}{\mathrm{d} t}$ denotes the operator of covariant derivation along $q(t)$ canonically associated with the riemannian metric of $Q$.

The well-posedness of the dynamics is given by Cauchy-Lipschitz theorem. We aim at extending this well-posedness result in the case where perfect unilateral constraints are added to the system. Hence, we consider some unilateral constraints defined by $n$ smooth functions $\varphi_{i}: Q \rightarrow \mathbb{R}$. Any admissible configuration of the system is supposed to belong to the set $A$ defined by formula (3). For all admissible configuration $q$ in $A$, we introduce:

- the set $J(q)$, defined by formula (4), of all active constraints in the configuration $q$,

- the closed convex cone $V(q)$, defined by formula (5), of all admissible right velocities in the configuration $q$,

- $N^{*}(q)$ and $N(q)$, which are the polar cones of $V(q)$, respectively for the duality $\left\langle T_{q} Q, T_{q}^{*} Q\right\rangle$ and for the euclidean structure of $T_{q} Q . N^{*}(q)$ is defined by formula (6).

The functions $\varphi_{i}$ are supposed to be functionally independent in the sense that, for all admissible configuration $q \in A$, the $\left(\mathrm{d} \varphi_{i}(q)\right)_{i \in J(q)}$ are linearly independent in $T_{q}^{*} Q$. In the case where the configuration space is $\mathbb{R}^{d}$, it is known $[1,2]$ that the motion of the system is to be searched in the class of all functions of time, taking values in $\mathbb{R}^{d}$, admitting a right velocity at each instant and such that the right velocity is a function of time with locally bounded variation. Each function $v$ with locally bounded variation is classically associated with its Stieljes measure which will be denoted by $\mathrm{d} v$. For an arbitrary riemannian configuration space, this definition admits the following generalization. If $J$ is a real interval, we denote by $\operatorname{MMA}(J, Q)$ (motions with measure acceleration) the class of all locally absolutely continuous motions from $J$ into $Q$, admitting a right velocity at each instant and such that the components of the right velocity in any chart have locally bounded variation in time. Any motion of $\operatorname{MMA}(J, Q)$ admits left and right velocities, $\dot{q}^{-}(t)$ and $\dot{q}^{+}(t)$, at each instant. These velocities are equal except for some instants which are called impacts and are at most countable. The class $\operatorname{MMA}(J, Q)$ can also be defined intrinsically by use of parallel transport. The covariant derivative of the velocity along the curve which is defined for $C^{2}$ motions can be generalized to any motion of $\operatorname{MMA}(I, Q)$ by use of a measure which can be defined intrinsically and whose expression in any local chart is given by formula (7), where $\mathrm{d} t$ denotes Lebesgue measure and the $e^{i}(q)$, the vectors of the natural basis of $T_{4}^{*} Q$ associated with the considered chart. Now, the reaction efforts appear as a measure $R$ defined by formula (8). The fact that these reaction efforts are of perfect contact type is expressed by formula (9) whose precise meaning is, by convention, that there exist $n$ nonpositive real measures $\lambda_{i}$ such that relations (10) hold. It is well-known that the equation of motion (9) is unable to determine the motion by itself and one has to add an impact constitutive equation. Following Moreau [2], we shall write the impact constitutive equation under the form (11), where $\operatorname{Proj}_{q}$ denotes the operator of orthogonal projection (for the scalar product of $T_{q} Q$ ) on a closed convex cone and $e$, an arbitrary function from $T Q$ into $[0,1]$, called restitution coefficient.

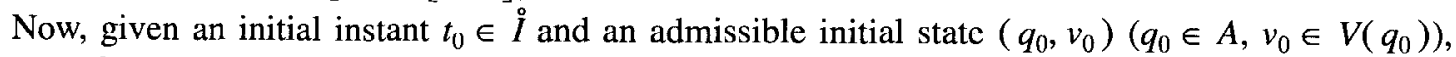
we can formulate the general evolution problem associated with the dynamics of discrete mechanical system with perfect unilateral constraints. 
PROBLEM $\mathscr{P}$. - Find $T \in \bar{I} \cup\{+\infty\}, T>t_{0}$ and $q \in M M A\left(\left[t_{0}, T[; Q)\right.\right.$ such that relations (12), (13), (14) and (15) hold.

Schatzman [1] gives a striking elementary example where, although all the data have regularity $C^{\infty}$, there are at least two solutions for this evolution problem. In the sequel, we assume that the riemannian manifold $Q$ is analytic, as well as the functions $\varphi_{i}$ and $f$. No regularity hypothesis is made on the restitution coefficient $e$. Under these assumptions, we have the following results.

THEOREM 2. - There is local existence and uniqueness of solution of problem $\mathscr{P}$ in the sense that:

- there exists a solution $(T, q)$ of problem $\mathscr{P}$;

- if $\left(T_{1}, q_{1}\right)$ and $\left(T_{2}, q_{2}\right)$ are two solutions of problem $\mathscr{P}$, then :

$$
\exists T, t_{0}<T \leq \min \left\{T_{1}, T_{2}\right\}, \quad q_{1 \mid\left[t_{0}, T[\right.}=q_{2 \mid\left[t_{0}, T[\right.}
$$

Then, a standard argument yields:

COROLlary 3. - Problem $\mathscr{P}$ admits a unique maximal solution $\left(T_{m}, q_{m}\right)\left(t_{0}<T_{m} \leq+\infty\right)$ in the sense that if $(T, q)$ denotes an arbitrary solution of problem $\mathscr{P}$, then:

$$
T \leq T_{m} \quad \text { and } \quad q=q_{m \mid\left[t_{0}\right.}, T[
$$

We shall say that the maximal solution of problem $\mathscr{P}$ is global if it is defined on $I \cap\left[t_{0},+\infty[\right.$

THEOREM 4. - Assume that the configuration space $Q$ is a complete riemannian manifold and that the efforts mapping $f$ admits the estimate:

$$
\begin{gathered}
\|f(q, v ; t)\|_{q} \leq l(t)\left(1+d\left(q, q_{0}\right)+\|v\|_{q}\right), \\
\forall(q, v) \in T Q, \text { for } \mathrm{d} t-\text { a.a. } t \in I \cap\left[t_{0},+\infty[\right.
\end{gathered}
$$

where $l(t)$ is a (necessarily nonnegative) function of $L_{\mathrm{loc}}^{1}(\mathbb{R} ; \mathbb{R})$. Then, the maximal solution of problem $\mathscr{P}$ is global.

'The detailed proof of these results is to be found in a forthcoming paper. In this reference, the continuous dependence of the solution on initial conditions is also studied, as well as the numerical computation of the solution.

\section{Introduction}

On considère un système de corps rigides soumis à des efforts intérieurs ct extéricurs ainsi qu'à des liaisons bilatérales scléronomes (ne dépendant pas explicitement du temps) et parfaites. La formulation lagrangiennc de la dynamique d'un tcl système fait intervenir une variété, dite de configuration, munie d'une structure riemannienne et conduit à une équation différentielle d'ordre deux sur cette variété. Si l'on se donne en plus la configuration ct la vitcsse du systc̀me en un instant initial, le théorème de Cauchy-Lipschitz garantit alors l'existence et l'unicité du mouvement du système sur un intervalle de temps maximal, moycnnant ccrtaines hypothèses de régularité sur les données.

Il arrive fréquemment que l'on doive en plus écrire des conditions de non-pénétration des solides entre eux ou des solides dans un obstacle fixe. Ces conditions se manifestent par des liaisons unilatérales que l'on supposera ici scléronomes et parfaites (les efforts de réaction ne travaillent pas dans toute vitesse virtuelle compatible avec le maintien de la liaison). La formulation générale de la dynamique des systèmes discrets avec liaisons unilatérales parfaites a retenu particulièrement l'attention de la communauté scientifique durant les 20 dernières annćes. Suivant Schatzman [1] et Moreau 
[2], on formule les équations de la dynamique en faisant intervenir la classe des mouvements dont l'accélération est une mesure vectorielle par rapport au temps. En fait, cette notion est ici convenablement adaptée au cas des variétés pour plus de généralité. On obtient ainsi une formulation générale du problème d'évolution correspondant. On s'intéresse au problème de l'existence et de l'unicité de la solution. Des résultats très partiels sur ces questions ont été obtenus par Schatzman [1], Percivale [3, 4] et Monteiro-Marques [5]. On trouve dans Schatzman [1] un exemple frappant de non-unicité dans un cas élémentaire où toutes les données sont de classe $C^{\infty}$. Dans cette note, on généralise un résultat de Percivale [3] démontré dans un cas très particulier et l'on obtient existence et unicité de la solution au problème d'évolution général pourvu que les données soient analytiques.

\section{Formulation générale du problème d'évolution}

DÉFINITION 1. - On appelle système mécanique discret la donnée de :

- Une variété régulière, séparée, connexe $Q$, dite espace de configuration du système. Sa dimension $d$ est le nombre de degrés de liberté du système. Son fibré tangent TQ est l'espace des phases du système. Le fibré cotangent sera noté $T^{*} Q$ et les projections naturelles, $\Pi_{Q}$ et $\Pi_{Q}^{*}$. Pour désigner un élément de $T Q$ (un état du système), on utilisera la notation redondante $(q, v)$ où $q=\Pi_{Q}(q, v)$.

- Une métrique riemannienne sur $Q$ notée $(., \text {. })_{q}$. L'application:

$$
K \begin{cases}T Q & \rightarrow \mathbb{R}^{+} \\ (q, v) & \mapsto \frac{1}{2}(v, v)_{q}=\frac{1}{2}\|v\|_{q}^{2}\end{cases}
$$

est appelée énergie cinétique du système.

- Un intervalle réel $I$ et une application régulière $f: T Q \times I \rightarrow T^{*} Q$ telle que :

$$
\forall(q, v) \in T Q, \quad \forall t \in I, \quad \Pi_{Q}^{*}(f(q, v ; t))=\Pi_{Q}(q, v)=q
$$

L'appplication $f$ est appelée puissance virtuelle des efforts intérieurs, extérieurs et d'inertie. On notera $\langle., .\rangle_{q}$ le produit de dualité local entre $T_{q} Q$ et $T_{q}^{*} Q$, ainsi que $\mathrm{b}$ (et $\#=\mathrm{b}^{-1}$ son inverse) l'isomorphisme de fibrés vectoriels de $T Q$ vers $T^{*} Q$ canonniquement associé à la métrique.

En l'absence de données supplémentaires, le Principe fondamental de la dynamique exige que tout mouvement du système soit au moins de classe $C^{2}$ et vérifie :

$$
\forall t, \quad \mathrm{~b} \frac{\mathrm{D} \dot{q}(t)}{\mathrm{d} t}=f(q(t), \dot{q}(t) ; t)
$$

où $\frac{\mathrm{D}}{\mathrm{d} t}$ désigne la dérivation covariante le long de la courbe $q(t)$.

On impose maintenant au système des liaisons unilatérales définies par $n$ fonctions régulières $\varphi_{i}: Q \rightarrow \mathbb{R}$ de sorte que toute configuration du système doive appartenir à l'ensemble des configurations admissibles défini par :

$$
A=\left\{q \in Q ; \quad \forall i \in\{1,2, \cdots, n\}, \quad \varphi_{i}(q) \leq 0\right\}
$$

Il est commode d'introduire, pour toute configuration $q \in A$, l'ensemble $J(q)$ des liaisons actives :

$$
J(q)=\left\{i \in\{1,2, \cdots-n\} ; \quad \varphi_{i}(q)=0\right\}
$$

On fait l'hypothèse que les fonctions $\varphi_{i}$ sont fonctionnellement indépendantes au sens où, pour toute configuration admissible $q \in A$, la partie $\left(\mathrm{d} \varphi_{i}(q)\right)_{i \in J(q)}$ est supposée libre dans $T_{q}^{*} Q$. On désigne par $V(q)$ le cône des vitesses à droite admissibles dans la configuration $q$ :

$$
V(q)=\left\{v \in T_{q} Q ; \quad \forall i \subset J(q), \quad\left\langle\mathrm{d} \varphi_{i}(q), v\right\rangle_{q} \leq 0\right\}
$$


et par $N^{*}(q)$ son cône polaire dans la dualité $\left\langle T_{q} Q, T_{q}^{*} Q\right\rangle$ :

$$
N^{*}(q)=\left\{\sum_{i=1}^{n} \lambda_{i} \mathrm{~d} \varphi_{i}(q) ; \quad \forall i \in J(q), \quad \lambda_{i} \geq 0, \quad \forall i \notin J(q), \quad \lambda_{i}=0\right\}
$$

Le cône polaire de $V(q)$ pour la structure euclidienne de $T_{q} Q$ est $N(q)=\#\left(N^{*}(q)\right)$.

Dans le cas où l'espace de configuration est $\mathbb{R}^{d}$ euclidien, il est classique $[1,2]$ que les mouvements du système soient à chercher dans la classe des fonctions du temps à valeur dans $\mathbb{R}^{d}$, admettant une vitesse à droite à chaque instant et telles que la vitesse à droite soit une fonction localement à variation bornée. À toute fonction localement à variation bornée $v$, on associe alors classiquement sa mesure de Stieljes notée $\mathrm{d} v$. On généralise cette notion pour un espace de configuration riemannien quelconque $Q$ de la manière suivante. Si $J$ est un intervalle réel, $\operatorname{MMA}(J, Q)$ (mouvements à accélération mesure) désigne la classe des courbes localement absolument continues de $J$ dans $Q$, admettant une vitesse à droite à tout instant et telles que la vitesse à droite ramenée dans le plan tangent $T_{q_{0}} Q$ en un point arbitraire $q_{0}$ de la courbe par transport parallèle le long de la courbe, soit une fonction localement à variation bornée à valeur dans $T_{q_{0}} Q$. De manière équivalente, on pourrait aussi définir la classe $M M A(J, Q)$ en requérant que les composantes de la vitesse à droite dans toute carte locale soient des fonctions numériques localement à variation bornée. Tout mouvement $q(t)$ dans $M M A(J, Q)$ admet alors des vitesses à gauche et à droite, $\dot{q}^{-}(t)$ et $\dot{q}^{+}(t)$ à chaque instant. Ces vitesses coïncident sauf pour des instants au plus dénombrables appelés impacts. La dérivée covariante de la vitesse à droite (ou à gauche) se généralise en une mesure pouvant être définie intrinsèquement et dont l'expression dans une carte locale est donnée par :

$$
\mathrm{bD} \dot{q}^{+}=\left(\mathrm{d} \frac{\partial K\left(q(t), \dot{q}^{+}(t)\right)}{\partial \dot{q}^{+i}}-\frac{\partial K\left(q(t), \dot{q}^{+}(t)\right)}{\partial q^{i}} \mathrm{~d} t\right) \mathrm{e}^{i}(q(t))
$$

où d $t$ est la mesure de Lebesgue et les $\mathrm{e}^{i}(q)$ les vecteurs de la base naturelle de $T_{q}^{*} Q$ associée à la carte considérée.

Les efforts de réaction apparaissent alors comme une mesure $R$ définie par :

$$
R=\mathrm{b} D \dot{q}^{+}-f\left(q(t), \dot{q}^{+}(t) ; t\right) \mathrm{d} t
$$

Le fait que les efforts de réaction soient de type contact parfait s'écrit alors :

$$
R=\mathrm{bD} \dot{q}^{+}-f\left(q(t), \dot{q}^{+}(t) ; t\right) \mathrm{d} t \in-N^{*}(q(t))
$$

ce qui, par convention, signifie précisément : il existe $n$ mesures réelles négatives $\lambda_{i}$ telles que :

$$
R=\sum_{i=1}^{n} \lambda_{i} \mathrm{~d} \varphi_{i}(q(t)) \quad \text { et } \quad \text { Supp } \lambda_{i} \subset\left\{t ; \varphi_{i}(q(t))=0\right\} \quad \forall i
$$

Il est bien classique que cette équation du mouvement ne détermine pas à elle seule le mouvement du système : il faut lui adjoindre une équation constitutive des impacts. Suivant Moreau [2], on écrira :

$$
\forall t, \quad \dot{q}^{+}=\operatorname{Proj}_{q}\left(\dot{q}^{-} ; V(q)\right)-e\left(q, \dot{q}^{-}\right) \operatorname{Proj}_{q}\left(\dot{q}^{-} ; N(q)\right)
$$

où $\operatorname{Proj}_{q}$ est l'opérateur de projection orthogonale (pour le produit scalaire de $T_{q} Q$ ) sur un cône convexe fermé et $e$ une fonction arbitraire de $T Q$ dans $[0,1]$ appcléc coefficient de restitution (des vitesses normales).

On est maintenant en mesure de formuler le problème d'évolution associé de manièrc gćnćralc à la dynamique des systèmes mécaniques discrets en présence de liaisons unilatérales parfaites. On se

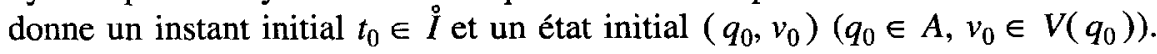


PROBLÈME $\mathscr{P} .-$ Trouver $T \in \bar{I} \cup\{+\infty\}, T>t_{0}$ et $q \in \operatorname{MMA}\left(\left[t_{0}, T[; Q)\right.\right.$ tels que :

$-\left(q\left(t_{0}\right), \dot{q}^{+}\left(t_{0}\right)\right)=\left(q_{0}, v_{0}\right)$

- $\forall t \in\left[t_{0}, T[\quad q(t) \in A\right.$

- $R=\mathrm{bD} \dot{q}^{+}-f\left(q(t), \dot{q}^{+}(t) ; t\right) \mathrm{d} t \in-N^{*}(q(t))$

- $\forall t \in] t_{0}, T\left[\quad \dot{q}^{+}=\operatorname{Proj}_{q}\left(\dot{q}^{-} ; V(q)\right)-e\left(q, \dot{q}^{-}\right) \operatorname{Proj}_{q}\left(\dot{q}^{-} ; N(q)\right)\right.$

\section{Existence et unicité de la solution}

On suppose ici que la variété riemannienne $Q$ est analytique ainsi que les fonctions $\varphi_{i}$ et $f$. Aucune hypothèse de régularité n'est faite sur la fonction $e$. On annonce alors les résultats suivants :

THÉORÈME 2. - Il y a existence et unicité locale de la solution au problème $\mathscr{P}$ au sens où :

- il existe une solution $(T, q)$ pour le problème $\mathscr{P}$,

- si $\left(T_{1}, q_{1}\right)$ et $\left(T_{2}, q_{2}\right)$ sont deux solutions du problème $\mathscr{P}$, alors :

$$
\exists T, \quad t_{0}<T \leq \min \left\{T_{1}, T_{2}\right\}, \quad q_{1 \mid\left[t_{0}, T[\right.}=q_{2 \mid\left[t_{0}, T\right]}
$$

Un argument standard donne alors :

COROllaire 3. - Le problème $\mathscr{P}$ admet une solution maximale unique $\left(T_{m}, q_{m}\right)\left(t_{0}<T_{m} \leq+\infty\right)$ au sens où si $(T, q)$ désigne une solution arbitraire du problème $\mathscr{P}$, alors :

$$
T \leq T_{m} \text { et } q=q_{m \mid\left[t_{0}, T[\right.}
$$

On dira que la solution maximale du problème $\mathscr{P}$ est globale si elle est définie sur $I \cap\left[t_{0},+\infty[\right.$.

THÉORÈME 4. - Supposons que la variété des configurations $Q$ soit une variété riemannienne complète et, qu'en outre, l'application f admette l'estimation :

$$
\begin{gathered}
\|f(q, v ; t)\|_{q} \leq l(t)\left(1+d\left(q, q_{0}\right)+\|v\|_{q}\right), \quad \forall(q, v) \in T Q, \\
\text { pour } \mathrm{d} t-\text { presque tout } t \in I \cap\left[t_{0},+\infty[,\right.
\end{gathered}
$$

où $l(t)$ est une fonction (necessairement positive) de $L_{\text {loc }}^{1}(\mathbb{R} ; \mathbb{R})$. Alors, la solution maximale du problème $\mathscr{P}$ est globale.

La démonstration détaillée de ces résultats est donnée dans un article à venir. Dans cette référence, on étudie en outre la dépendance continue de la solution vis-à-vis des conditions initiales, ainsi que la convergence de l'algorithme introduit par Moreau [2] pour le calcul des solutions.

\section{Références bibliographiques}

[1] Schatzman M., A class of nonlinear differential equations of second order in time, Nonlinear Anal., Theor., Meth. Appl. 2 (1978) 355-373.

[2] Moreau J.J., Standard inelastic shocks and the dynamics of unilateral constraints, in: Del Piero G., Maceri F. (Eds), Unilateral problems in structural analysis, Springer-Verlag, Wien, New-York, 1983, pp. 173-221.

[3] Percivale D., Uniqueness in the elastic bounce problem, I, J. Differ. Equations 56 (1985) 206-215.

[4] Percivale D., Uniqueness in the elastic bounce problem, II, J. Differ. Equations 90 (1991) 304-315.

[5] Monteiro-Marques M.D.P., Differential Inclusions in Nonsmooth Mechanical Problems, Birkhaüser, Basel, Boston, Berlin, 1993. 